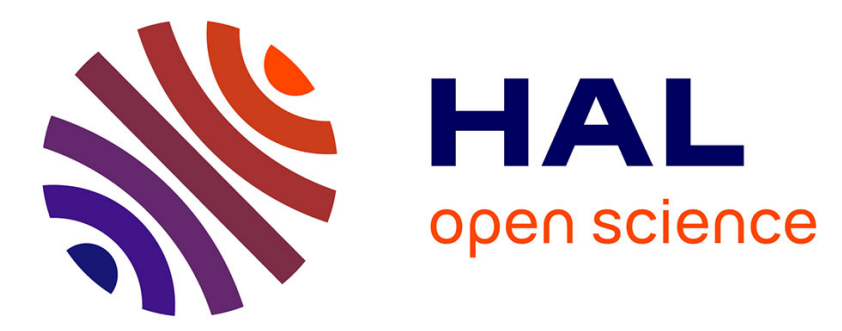

\title{
Étude des premiers niveaux excités de 6Li
}

\author{
I. Linck, R. Bilwes, L. Kraus, R. Seltz, D. Magnac-Valette
}

\section{To cite this version:}

I. Linck, R. Bilwes, L. Kraus, R. Seltz, D. Magnac-Valette. Étude des premiers niveaux excités de 6Li. Journal de Physique, 1969, 30 (1), pp.17-28. 10.1051/jphys:0196900300101700 . jpa-00206758

\section{HAL Id: jpa-00206758 https://hal.science/jpa-00206758}

Submitted on 1 Jan 1969

HAL is a multi-disciplinary open access archive for the deposit and dissemination of scientific research documents, whether they are published or not. The documents may come from teaching and research institutions in France or abroad, or from public or private research centers.
L'archive ouverte pluridisciplinaire HAL, est destinée au dépôt et à la diffusion de documents scientifiques de niveau recherche, publiés ou non, émanant des établissements d'enseignement et de recherche français ou étrangers, des laboratoires publics ou privés. 


\title{
ÉTUDE DES PREMIERS NIVEAUX EXGITÉS DE ${ }^{6} \mathrm{Li}$
}

\author{
Par I. LINGK $\left({ }^{1}\right)$, R. BILWES, L. KRAUS, R. SELTZ et D. MAGNAG-VALETTE, \\ Groupe des Basses Énergies, Centre de Recherches Nucléaires, Strasbourg-Cronenbourg.
}

(Reçu le 29 juillet 1968.)

\begin{abstract}
Résumé. - Les auteurs donnent un nouveau schéma des cinq premiers niveaux excités de lithium 6 déduit de l'étude spectroscopique de la réaction ${ }^{7} \mathrm{Li}\left({ }^{3} \mathrm{He}, \alpha\right)^{6} \mathrm{Li}$ entre 1 et $12 \mathrm{MeV}$ d'énergie incidente et de l'interprétation de la diffusion résonnante ${ }^{4} \mathrm{He}+\mathrm{d}$ dans le formalisme de la matrice $S$. Ils présentent un essai d'interprétation du mécanisme de la première réaction dans l'approximation de Born en ondes distordues (D.W.B.A.).
\end{abstract}

\begin{abstract}
A new diagram of the five first excited levels of ${ }^{6} \mathrm{Li}$ is deduced from the spectroscopic study of the ${ }^{7} \mathrm{Li}\left({ }^{3} \mathrm{He}, \alpha\right)^{6} \mathrm{Li}$ reaction between $1 \mathrm{MeV}$ and $12 \mathrm{MeV}$ laboratory energy and from the $S$-matrix formalism interpretation of the ${ }^{4} \mathrm{He}+\mathrm{d}$ resonant scattering. The angular distributions and excitation curves of $\alpha$-particles in the first reaction are tentatively interpreted by direct processes in the D.W.B.A. theory.
\end{abstract}

Introduction. - Les caractéristiques des premiers niveaux de ${ }^{6} \mathrm{Li}$ sont des données expérimentales déterminantes du point de vue théorique [1]. Elles conditionnent en effet de façon très restrictive la valeur des paramètres utilisés par les théoriciens des noyaux de la couche $p$ ou, si elles ne peuvent entrer dans une théorie générale, font attribuer au lithium 6 des propriétés particulières que les théoriciens tentent de justifier.

Quand Inglis [1] applique au lithium 6 sa théorie du couplage LS et du couplage intermédiaire pour les noyaux de la couche $1 p$, la triade de masse 6 est encore peu connue. La valeur du paramètre $a / K \simeq 1,3$ rend bien compte des trois premiers états de ${ }^{6} \mathrm{Li}$, mais donne une énergie d'excitation trop grande pour le premier état de ${ }^{6} \mathrm{He}$. Inglis préfère utiliser $a / K \simeq 5$ pour ajuster cette donnée expérimentale; il est alors entraîné à faire une correction de structuration pour rendre compte de l'ordre des trois premiers niveaux de ${ }^{6} \mathrm{Li}$.

S. Cohen et D. Kurath [1], dans un calcul d'interaction effective pour les noyaux de la couche $p$, déterminent des paramètres moyens à partir des données expérimentales connues en 1959 [2] et concernant les noyaux de masse $A=8$ à 16 d'une part, $A=6$ à 16 de l'autre. L'accord obtenu entre les calculs et les valeurs expérimentales relatives aux six premiers niveaux de ${ }^{6} \mathrm{Li}$ est peu satisfaisant, que ces valeurs soient incluses ou non dans les programmes.

Goldhammer, Hill et Nachamkin [1] ont publié récemment un calcul analogue à celui de Cohen et

(1) Cet article donne l'essentiel de la thèse de Mme Isabelle Linck, soutenue le 2 mai 1968.
Kurath, mais qui tient compte d'une interaction à trois corps. Là encore, les valeurs expérimentales concernant les six premiers niveaux de ${ }^{6} \mathrm{Li}$ conditionnent les résultats des calculs. Or il existe plusieurs controverses entre les résultats expérimentaux qui concernent les premiers états inférieurs de lithium 6 .

Il nous a semblé intéressant de reprendre l'analyse de la réaction ${ }^{7} \mathrm{Li}\left({ }^{3} \mathrm{He}, \alpha\right)^{6} \mathrm{Li}$ et de la diffusion résonnante ${ }^{4} \mathrm{He}+\mathrm{d}$, qui ont tout particulièrement permis d'établir le schéma des niveaux de ${ }^{6} \mathrm{Li}$, utilisé jusqu'à présent par les théoriciens. Nous comparons nos résultats avec ceux déduits d'autres expériences et donnons en conclusion un nouveau schéma des six premiers niveaux de ${ }^{6} \mathrm{Li}$.

I. Réaction ${ }^{7} \mathbf{L i}\left({ }^{3} \mathrm{He}, \alpha\right)^{6} \mathbf{L i}$. - La réaction ${ }^{7} \mathrm{Li}\left({ }^{3} \mathrm{He}, \alpha\right){ }^{6} \mathrm{Li}$ permet d'atteindre les états de ${ }^{6} \mathrm{Li}$ quel que soit leur spin isobarique, dans un large domaine d'excitation grâce au bilan de $13,325 \mathrm{MeV}$.

Allen et collaborateurs [3] ont fait une étude de cette réaction entre 0,6 et $0,9 \mathrm{MeV}$ aux environs de $90^{\circ}$ et en ont déduit neuf niveaux dans le lithium 6 qui furent reportés dans les tables jusqu'en 1962 [2]. Ces résultats, en ce qui concerne les cinq premiers niveaux, confirmaient les calculs antérieurs d'Inglis qui, pour un couplage intermédiaire proche d'un couplage $\operatorname{LS}(a / K \simeq 1,3)$, prévoient la suite $\left(1^{+}, 0\right)$ $\left(3^{+}, 0\right)\left(0^{+}, 1\right)\left(2^{+}, 0\right)\left(1^{+}, 0\right)$ et $\left(2^{+}, 1\right)$, les deux derniers niveaux très proches l'un de l'autre et à $6 \mathrm{MeV}$ tandis que le $\left(2^{+}, 0\right)$ serait vers $4,5 \mathrm{MeV}$. Ils confirmaient d'autre part les résultats de Galonsky et collaborateurs [11] sur le triplet $D^{3+, 2+, 1+} T=0$ obtenus dans l'interprétation de la diffusion résonnante ${ }^{4} \mathrm{He}+\mathrm{d}$. 


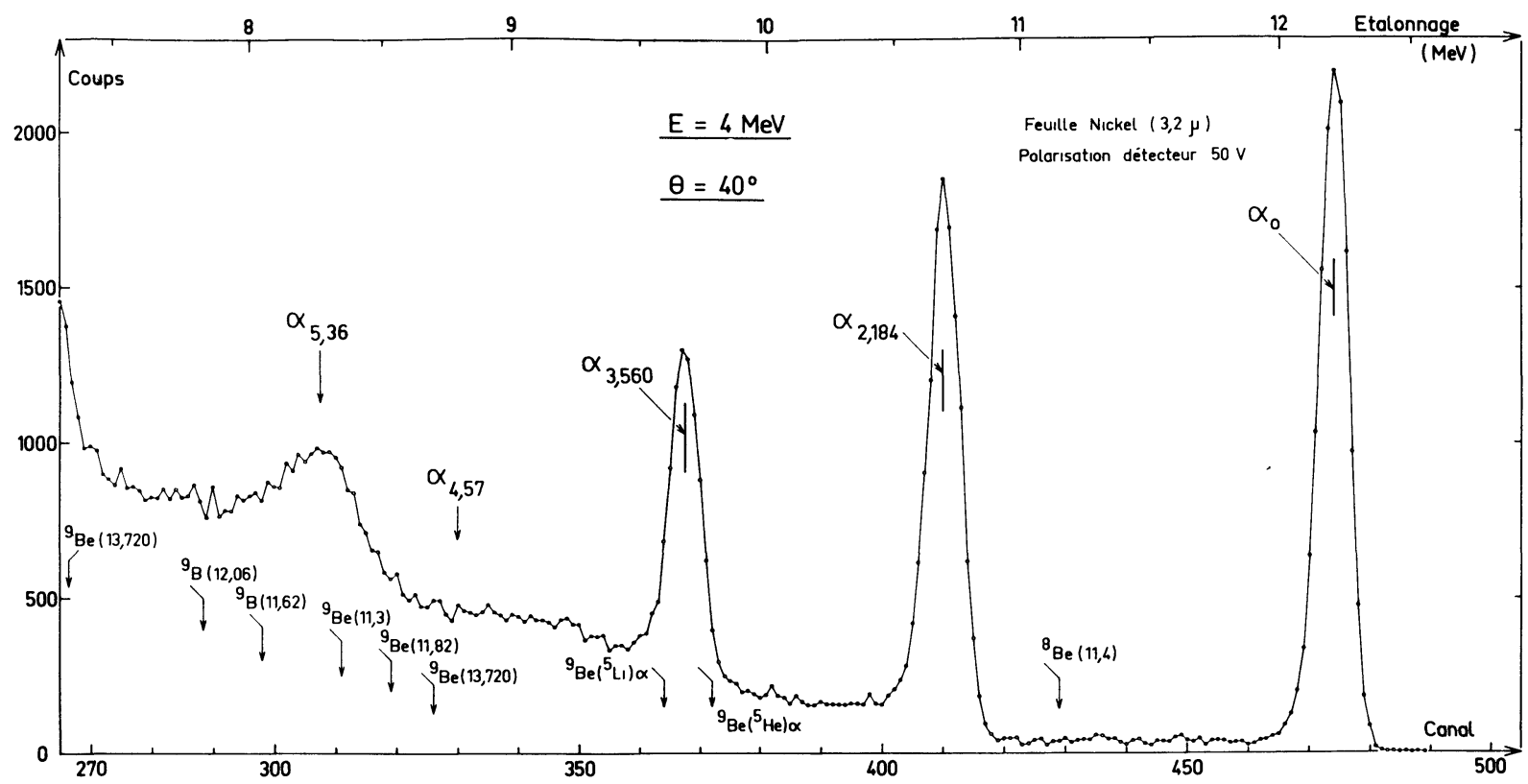

FIG. 1. - Spectre de la réaction ${ }^{7} \mathrm{Li}\left({ }^{3} \mathrm{He}, \alpha\right)^{6} \mathrm{Li}$

obtenu avec un détecteur à semiconducteurs muni d'un écran de nickel.

1. Résultats expérimentaux. - Une première étude effectuée en collaboration avec J. Linck [4] à l'aide d'un spectrographe magnétique de type Buechner ne nous a pas permis de retrouver certains des niveaux donnés par Allen et collaborateurs, notamment un état étroit à $5,35 \mathrm{MeV}$ et deux états larges à $4,52 \mathrm{MeV}$ et $5,5 \mathrm{MeV}$. Nous avons complété cette étude, tant du point de vue énergétique que du point de vue angulaire, afin de vérifier si l'absence des états prévus théoriquement par Inglis et confirmés par l'étude de Galonsky et collaborateurs pouvait être attribuée au mécanisme de la réaction. Nous avons pour cela préféré au spectrographe magnétique des détecteurs à semiconducteurs moins résolvants et, par suite, mieux appropriés à la mise en évidence d'états larges dans une réaction de faible section efficace.

A l'aide de huit détecteurs à semiconducteurs disposés entre $20^{\circ}$ et $165^{\circ}$, nous avons étudié la réaction ${ }^{7} \mathrm{Li}\left({ }^{3} \mathrm{He}, \alpha\right)^{6} \mathrm{Li}$ entre 1 et $12 \mathrm{MeV}$ d'énergie incidente. Les cibles ont été obtenues par évaporation de ${ }^{7} \mathrm{Li}$ enrichi sur du formvar. Leur épaisseur varie entre 50 et $100 \mathrm{keV}$ pour des $\alpha$ de $8,78 \mathrm{MeV}$. La profondeur des détecteurs a été choisie de manière à ne pas absorber complètement les protons, deutérons et tritons des réactions compétitives très exothermiques. On n'a ainsi que des particules $\alpha$ dans la région des spectres correspondant à une énergie d'excitation inférieure à $6 \mathrm{MeV}$ dans le lithium 6 . Des écrans de nickel de 1 à 3 microns ont permis d'éliminer de cette région le pic très important de reculs de ${ }^{6} \mathrm{Li}$ dans son état fondamental. La difficulté de l'étude se réduit à la contamination par ${ }^{16} \mathrm{O}\left({ }^{3} \mathrm{He}, \alpha_{0}\right){ }^{15} \mathrm{O}$ et par des fonds continus dus à la désexcitation des niveaux fortement excités de ${ }^{9} \mathrm{~B},{ }^{9} \mathrm{Be},{ }^{8} \mathrm{Be}$ et ${ }^{6} \mathrm{Li}$ dont l'importance augmente avec l'énergie incidente.

La figure 1 présente un des nombreux spectres étudiés. Les résultats déduits de ces spectres confirment ceux obtenus à l'aide du spectrographe magnétique, c'est-à-dire :

- Trois états de faible largeur à $0 ; 2,18$ et $3,57 \mathrm{MeV}$ avec $\left(J^{+}, T\right)=\left(1^{+}, 0\right)\left(3^{+}, 0\right)$ et $\left(0^{+}, 1\right)$ respectivement;

- Aucun état large dans la région de $4,5 \mathrm{MeV}$ d'excitation et au-dessus;

- Un seul état à $5,36 \mathrm{MeV}$ avec une largeur de $540 \mathrm{keV}$. Ces résultats ont d'autre part été trouvés par L. Cocke [5].

Le tableau I résume les résultats obtenus sur les niveaux de ${ }^{6} \mathrm{Li}$ à partir de la réaction ${ }^{7} \mathrm{Li}\left({ }^{3} \mathrm{He}, \alpha\right)^{6} \mathrm{Li}$.

2. Discussion. - Nous avons comparé ces résultats avec ceux parus jusqu'à maintenant dans la littérature et obtenus à partir d'autres réactions (tableaux II et III). Ces résultats diffèrent beaucoup les uns des autres, mais on peut relever des particularités communes à plusieurs auteurs.

a) L'état entre 4 et $5 \mathrm{MeV}$ n'est signalé en diffusion qu'à des énergies incidentes supérieures à $14 \mathrm{MeV}$ (même dans celles qui trient le spin isobarique $T=0)[6,7,8]$. Il n'a jamais été trouvé à partir de ${ }^{6} \mathrm{Li}\left(\mathrm{d}, \mathrm{d}^{\prime}\right)^{6} \mathrm{Li}$.

Cet état n'est signalé dans aucune des réactions $(p, d),(d, t),\left({ }^{3} \mathrm{He}, \alpha\right)[5,10]$ si l'on excepte l'expérience d'Allen dont nous croyons avoir démontré la 
TABLEAU I

LISTE DES NIVEAUX DE ${ }^{6} \mathrm{Li}$ DANS LA RÉAGTION ${ }^{7} \mathrm{Li}\left({ }^{3} \mathrm{He}, \alpha\right)^{6} \mathrm{Li}$

par Allen [3], Cogke [5] et les auteurs [4] (et présent travail)

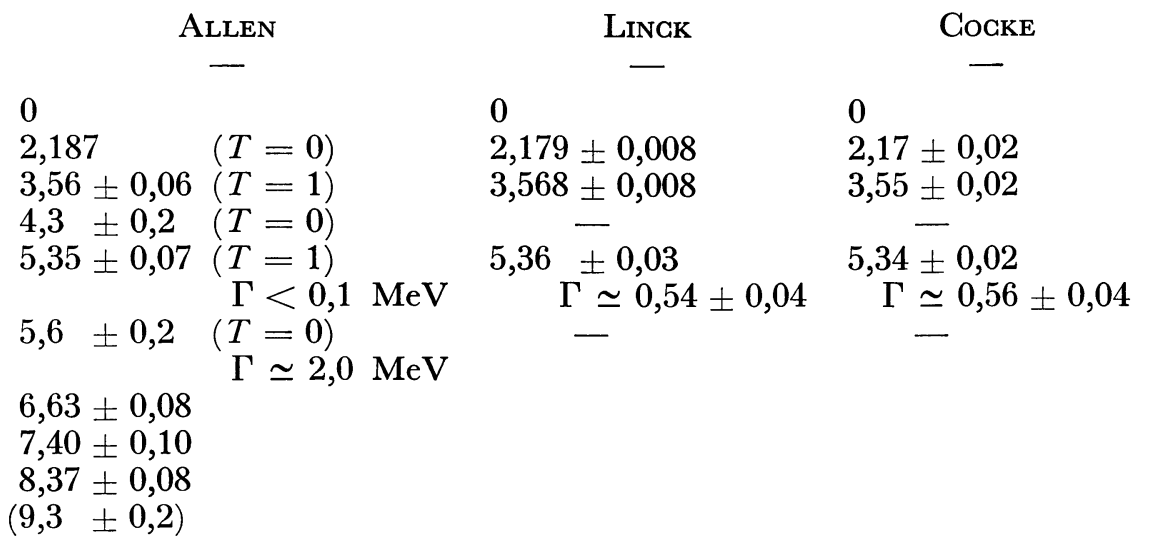

Toutes les énergies sont données en $\mathrm{MeV}$.

TABLEAU II

\begin{tabular}{|c|c|c|c|c|c|c|c|}
\hline \multirow{2}{*}{ RÉF. } & \multirow{2}{*}{$\begin{array}{c}\text { RÉAGTION } \\
-\end{array}$} & \multirow{2}{*}{$\begin{array}{c}E_{1} \\
\text { INGIDENTE } \\
-\end{array}$} & \multicolumn{4}{|c|}{ NIVEAUX OBSERVÉS } & \multirow{2}{*}{ REMARQUES } \\
\hline & & & $\underline{2,18}$ & $\underline{3,56}$ & $\begin{array}{c}\text { Entre } 4 \\
\text { et } 5 \mathrm{MeV} \\
\end{array}$ & $\begin{array}{c}\text { Entre } 5 \\
\text { et } 6 \mathrm{MeV} \\
-\end{array}$ & \\
\hline Br57a [7] & ${ }^{6} \mathrm{Li}\left(\mathrm{dd}^{\prime}\right)$ & $14 \mathrm{MeV}$ & 2,18 & I & Rien & IIIIIIIIIIIIIIII & S-SM \\
\hline $\mathrm{Ha} 60 \mathrm{a}[10]$ & ${ }^{6} \mathrm{Li}\left(\mathrm{dd}^{\prime}\right)$ & 14,8 & 2,18 & I & Rien & IIIIIIIIIIIIIII & S-SM \\
\hline Wa56 [6] & ${ }^{6} \operatorname{Li}\left(\alpha \alpha^{\prime}\right)$ & 31,5 & 2,18 & I & 4,52 & ІІІІІІІІІІІІІІІІ & $\begin{array}{l}\text { Description peu ex- } \\
\text { plicite des résultats }\end{array}$ \\
\hline Gr64 [6] & ${ }^{6} \mathrm{Li}\left(\alpha \alpha^{\prime}\right)$ & 23,8 & 2,18 & I & 4,52 & ІІІІІІІІІІІІІІІІ & $\begin{array}{l}\text { Pas de spectres don- } \\
\text { nés }\end{array}$ \\
\hline Br57a [7] & ${ }^{6} \mathrm{Li}\left(\mathrm{pp}^{\prime}\right)$ & 14 & 2,188 & 3,56 & Rien & IIIIIIIIIIIIIII & S-SM \\
\hline Ha65 [7] & ${ }^{6} \mathrm{Li}\left(\mathrm{pp}^{\prime}\right)$ & 185 & 2,2 & 3,6 & $\begin{array}{l}\text { Bosse } \\
\text { large }\end{array}$ & & S-faible résolution \\
\hline $\operatorname{Ma} 68$ [7] & ${ }^{6} \mathrm{Li}\left(\mathrm{pp}^{\prime}\right)$ & 50 & 2,14 & 3,49 & $\begin{array}{l}4,03 \\
4,45\end{array}$ & 5,28 & $\begin{array}{r}\text { En contradiction avec } \\
\mathrm{d}+\alpha \text { par le même } \\
\text { auteur }(\mathrm{Ma68})[13]\end{array}$ \\
\hline Bo65g [8] & ${ }^{6} \mathrm{Li}\left(\mathrm{nn}^{\prime}\right)$ & 14 & 2,18 & 3,56 & $4 \mathrm{MeV}$ & 5 et 5,5 & $\begin{array}{l}\text { Les pics attribués sont } \\
\text { décalés }\end{array}$ \\
\hline Br57a [7] & ${ }^{9} \mathrm{Be}(\mathrm{p}, \alpha){ }^{6} \mathrm{Li}$ & 14 & 2,188 & 3,560 & Rien & IIIIIIIIIIIIIIII & S-SM \\
\hline Gr63d [9] & ${ }^{9} \mathrm{Be}(\mathrm{p}, \alpha){ }^{6} \mathrm{Li}$ & 10 & 2,18 & 3,55 & 4,4 & 5,32 & $\begin{array}{l}\text { Pas d'étude des conti- } \\
\text { nuums sous-jacents }\end{array}$ \\
\hline $\begin{array}{l}\text { I } \\
\text { IIIII } \\
\text { SM } \\
\mathrm{S}\end{array}$ & $\begin{array}{l}\text { rographe } \\
\text { tres. }\end{array}$ & égion. & & & & & \\
\hline
\end{tabular}


TABLEAU III

\begin{tabular}{|c|c|c|c|c|c|c|c|}
\hline \multirow{2}{*}{ RÉF. } & \multirow{2}{*}{$\begin{array}{c}\text { RÉAGTION } \\
-\end{array}$} & \multirow{2}{*}{$\begin{array}{c}E_{1} \text { INGIDENTE } \\
-\end{array}$} & \multicolumn{4}{|c|}{ Niveaux OBSERVÉS } & \multirow{2}{*}{ RemarQUes } \\
\hline & & & $\begin{array}{c}2,18 \\
-\end{array}$ & $\begin{array}{c}3,56 \\
-\end{array}$ & $\begin{array}{c}\text { Entre } 4 \\
\text { et } 5 \mathrm{MeV} \\
-\end{array}$ & $\begin{array}{l}\text { Entre } 5 \\
\text { et } 6 \mathrm{MeV} \\
-\end{array}$ & \\
\hline Ku67 [19] & ${ }^{7} \mathrm{Li}(\mathrm{pd})$ & 33,6 & 2,15 & 3,57 & Rien & 5,38 & S-SM \\
\hline $\begin{array}{l}\text { Le55 [19] } \\
\text { Ha60a [10] }\end{array}$ & ${ }^{7} \mathrm{Li}(\mathrm{dt})$ & 14,5 & 2,188 & 3,56 & Rien & $\begin{array}{c}\text { IIIIIIIIIIIIIIII } \\
5,40\end{array}$ & S-DA-SM \\
\hline Co67 [5] & ${ }^{7} \mathrm{Li}\left({ }^{3} \mathrm{He}, \alpha\right)$ & 0,9 à 12 & 2,188 & 3,56 & Rien & 5,34 & SM-J \\
\hline Li63c [4] & ${ }^{7} \mathrm{Li}\left({ }^{3} \mathrm{He}, \alpha\right)$ & De 1 à 5 & 2,179 & 3,568 & Rien & 5,47 & S-SM \\
\hline $\begin{array}{l}\text { Ce travail } \\
\qquad \begin{aligned} \text { IIIII } \\
\mathrm{SM} \\
\mathrm{S} \\
\mathrm{DA} \\
\mathrm{J}\end{aligned}\end{array}$ & $\begin{array}{l}{ }^{7} \mathrm{Li}\left({ }^{3} \mathrm{He}, \alpha\right) \\
\text { s d'étude de } \\
\text { ectrographe } \mathrm{n} \\
\text { ectres. } \\
\text { tribution ang } \\
\text { ictions. }\end{array}$ & $\begin{array}{l}\text { De } 1 \text { à } 12 \mathrm{MeV} \\
\text { région. } \\
\text { gnétique. } \\
\text { aire. }\end{array}$ & 2,18 & 3,56 & Rien & 5,36 & J-DA \\
\hline
\end{tabular}

non-reproductibilité. Dans la réaction ${ }^{9} \mathrm{Be}(\mathrm{p}, \alpha)^{6} \mathrm{Li}$ à $10 \mathrm{MeV}$ [9], seule une " bosse " très faible apparaît à l'endroit intéressé dans un cas où, justement, les fonds continus sont très importants.

Par contre, l'interprétation en matrice $R$ de la diffusion résonnante ${ }^{4} \mathrm{He}(\mathrm{d}, \mathrm{d}){ }^{4} \mathrm{He}$ nécessite un état $\left(2^{+}, 0\right)$ assez large dans cette région d'excitation $[11,12]$ à une exception près [13] où il est situé à $5,55 \mathrm{MeV}$. Cette dernière publication est d'ailleurs en contradiction avec celle publiée par un des deux auteurs sur ${ }^{6} \mathrm{Li}\left(\mathrm{p}, \mathrm{p}^{\prime}\right){ }^{6} \mathrm{Li}$ [7].

b) L'état vers $5,4 \mathrm{MeV}$ apparaît quand on dispose d'une énergie suffisante. Il est large et difficile à étudier parce que superposé à des fonds importants. La valeur de son spin isobarique est ambiguë. En effet :

- Son existence n'a pas été contrôlée dans les réactions $\left(\alpha, \alpha^{\prime}\right)$ et $\left(\mathrm{d}, \mathrm{d}^{\prime}\right)$ qui l'interdisent si $T=1$.

- Son spin isobarique a souvent été supposé nul à cause de l'interprétation en matrice $R$ de la diffusion résonnante ${ }^{4} \mathrm{He}(\mathrm{d}, \mathrm{d})^{4} \mathrm{He}$. Pourtant cette interprétation conduit à un niveau beaucoup plus large et dont l'énergie d'excitation, très différente suivant les auteurs $[11,12,13]$, est toujours supérieure à $5,40 \mathrm{MeV}$.

- D'autre part, il est dans la région où doit se trouver l'état analogue $T=1 \mathrm{du}$ premier état excité à $1,80 \mathrm{MeV}$ de ${ }^{6} \mathrm{He}$. Allen avait cru trouver dans cette région un niveau étroit auquel il attribuait un spin isobarique $T=1$, car le spin $T=0$, autorisant la décomposition en ${ }^{4} \mathrm{He}+\mathrm{d}$, aurait donné lieu à un état virtuel large. Or ce niveau étroit n'a été retrouvé par aucun auteur. D'autre part, un spin isobarique non nul n'exclurait pas pour autant la désexcitation par particules ni, par conséquent, une largeur notable
(540 keV pour le niveau de 5,36 MeV) : deux modes de décomposition sont en effet possible ${ }^{6} \mathrm{Li} \rightarrow{ }^{5} \mathrm{He}$ $+\mathrm{p}+0,71 \mathrm{MeV}$ et ${ }^{6} \mathrm{Li} \rightarrow \alpha+\mathrm{n}+\mathrm{p}+1,66 \mathrm{MeV}$.

Le niveau $T=1$ que nous cherchons dans le lithium 6 doit avoir le même spin et la même parité que le premier état excité de ${ }^{6} \mathrm{He}$. Cet état, de spin et parité $2^{+}$, situé à $1,797 \pm 0,025 \mathrm{MeV}$ [14], a une largeur de $113 \mathrm{keV}$ et trois modes de décomposition possibles : désexcitation par transition quadrupolaire électrique, tripartition et, à la limite, bipartition. Nous avons pu mettre en évidence [15] que la désexcitation par voie $\gamma$ est très peu probable $\left(\Gamma_{\lambda} / \Gamma_{\alpha} \leqslant 2 \times 10^{-6}\right)$ et que la tripartition $\alpha+2 \mathrm{n}$ entre pour $30 \%$ au moins dans la désexcitation de ${ }^{6} \mathrm{He}^{*}(1,80 \mathrm{MeV})$. L'inhibition de la désexcitation électromagnétique est toujours observée dans l'étude de l'hyperfragment ${ }_{\Lambda}^{7} \mathrm{He}$ [16], malgré la stabilité accrue de ${ }^{6} \mathrm{He}^{*}$ par rapport à l'émission de neutrons. La prépondérance de la désexcitation par tripartition a aussi été trouvée pour le premier état excité de ${ }^{6} \mathrm{Be}$ [17].

L'état excité de ${ }^{6} \mathrm{He}$, dont la configuration semble bien être celle d'un cœur $\alpha$ et de deux neutrons de spins antiparallèles dans la courbe $1 p$, ne diffère de l'état fondamental que par la valeur du moment orbital relatif total des deux nucléons extérieurs $(2$ au lieu de 0). Ces deux états sont excités de la même manière par la réaction ${ }^{7} \mathrm{Li}(\mathrm{t}, \alpha)^{6} \mathrm{He}$, excepté aux très basses énergies, et celui de $1,80 \mathrm{MeV}$ l'est même plus favorablement.

On ne voit donc pas pourquoi l'état analogue du premier état excité de ${ }^{6} \mathrm{He}:{ }^{6} \mathrm{Li}\left(2^{+}, T=1\right)$ ne serait pas excité par ${ }^{7} \mathrm{Li}\left({ }^{3} \mathrm{He}, \alpha\right)^{6} \mathrm{Li}$, alors que l'état $\left(0^{+}, T=1\right)$ analogue de l'état fondamental de ${ }^{6} \mathrm{He}$ est clairement observé. 
Il n'y a aucune raison pour ne pas admettre que l'état à $5,40 \mathrm{MeV}$ d'excitation dans ${ }^{6} \mathrm{Li}$ est l'analogue isobarique de l'état $\left(2^{+}, 1\right)$ à $1,80 \mathrm{MeV}$ dans ${ }^{6} \mathrm{He}$.

II. Diffusion résonnante ${ }^{4} \mathbf{H e}(\mathbf{d}, \mathbf{d})^{4} \mathrm{He}$. - Toutes les interprétations de la diffusion résonnante ${ }^{4} \mathrm{He}(\mathrm{d}, \mathrm{d}){ }^{4} \mathrm{He}$ dans le formalisme de la matrice $R[11,12,13]$ ont nécessité l'intervention du triplet $\mathrm{D}, T=0$. Les quantités caractéristiques de ces états (énergie d'excitation, largeur) sont très différentes selon les auteurs. Ces différences reflètent l'ambiguïté sur la détermination des paramètres qu'entraîne l'utilisation de la matrice $R$ surtout dans le cas d'un noyau très léger comme le lithium 6. D'autre part, tous les auteurs sont partis des résultats de la première étude réalisée par Galonsky et McEllistrem [3], pour le choix des déphasages initiaux en particulier.

1. Le formalisme DE LA MATrige $S$. - Le formalisme de la matrice $S$ proposé par Humblet et Rosenfeld permet une paramétrisation univoque de la section efficace d'une réaction en faisant de l'énergie $E$ une quantité complexe et en étudiant les propriétés analytiques de la matrice de diffusion dans le plan des énergies complexes.

Nous présentons ici les résultats obtenus à partir de l'interprétation des mesures expérimentales des auteurs précédents $[11,12,18]$ dans ce formalisme. Le détail des calculs est exposé plus explicitement par ailleurs [19].

L'élément de matrice $T_{\mathrm{c}^{\prime} \mathrm{c}}$ pour la transition de la voie d'entrée $c$ à une voie de sortie $c^{\prime}$ caractérisée par un moment angulaire total et une parité définis suivant les ondes à considérer est de la forme [20] :

$$
\begin{aligned}
& T_{\mathrm{c}^{\prime} \mathrm{c}}=\left(\Omega_{\mathrm{c}^{\prime}} \Omega_{\mathrm{c}}-1\right) \delta_{\mathrm{c}^{\prime} \mathrm{c}}+\Omega_{\mathrm{c}^{\prime}} \Omega_{\mathrm{c}}\left(k_{\mathrm{c}^{\prime}} k_{\mathrm{c}} \pi_{\mathrm{c}^{\prime}} \pi_{\mathrm{c}}\right)^{1 / 2} \times \ldots \\
& \times\left[Q_{\mathrm{c}^{\prime} \mathrm{c}}(E)-i \sum_{n} \frac{O_{\mathrm{c}^{\prime} n} O_{\mathrm{c} n}}{\left|K_{\mathrm{c}^{\prime} n} K_{\mathrm{c} n}\right|^{1 / 2}} \mathrm{e}^{i \bar{\xi}_{\mathrm{c}^{\prime} n} n} \frac{\Gamma_{\mathrm{c}^{\prime} n}^{1 / 2} \Gamma_{\mathrm{c} n}^{1 / 2} \mathrm{e}^{i \bar{\xi} \mathrm{c} n}}{E-E_{n}+i \frac{\Gamma_{n}}{2}}\right] .
\end{aligned}
$$

Le premier terme de cette expression tient compte de la diffusion par une sphère dure; il s'annule dans le cas d'une réaction.

Le deuxième terme est la somme d'un terme résonnant dont toutes les quantités affectées de l'indice $n$ sont prises à l'énergie de résonance $\mathscr{E}_{n}$ et d'un terme de « fond continu ».

L'énergie de résonance est $\mathscr{E}_{n}=E_{n}-i \Gamma_{n} / 2 . \Gamma_{n}$, la largeur totale du niveau résonnant, doit être égale à la somme des largeurs partielles $\Gamma_{\mathrm{c} n}$ à un facteur $q_{n}$ près, proche de $1 . \xi_{c n}$ est la phase réelle.

La pénétrabilité est de la forme :

$$
\pi_{\mathrm{c}}=\frac{1}{\left(F_{l}^{2}+G_{l}^{2}\right)_{r=b}}
$$

où $F_{l}$ et $G_{l}$ sont les fonctions coulombiennes usuelles.
On a d'autre part $\Omega_{\mathrm{c}}=\mathrm{e}^{i \Phi_{\mathrm{c}}}$ avec :

$$
\begin{gathered}
\Phi_{c}=-\operatorname{arctg}\left[\frac{F_{l}}{G_{l}}\right]_{r=b} \\
O_{c n}=G_{l}+i F_{l} .
\end{gathered}
$$

La section efficace différentielle qui a la même forme que dans le formalisme de la matrice $R$ [27], aux éléments de transition près, n'est linéaire en aucun de ses paramètres. L'ajustement des valeurs théoriques de la section efficace aux valeurs expérimentales a été réalisé à l'aide d'une méthode particulière de minimisation de $\chi^{2}$ appelée " recherche directe » [21], le seul paramètre fixé à l'avance étant le rayon coulombien $b$.

2. RÉsultats. - Un premier ajustement a été réalisé au voisinage de la résonance $D^{3+}$ à l'aide des éléments de matrice $T_{2}^{3+}$ (résonance + fond) et $T_{0}^{1+}$ (fond). L'accord est satisfaisant, mais il n'en est plus de même si l'on tient compte de ces seules contributions entre 3 et $12 \mathrm{MeV}$ (courbe $\mathrm{n}^{0} 1$ de la figure 2).

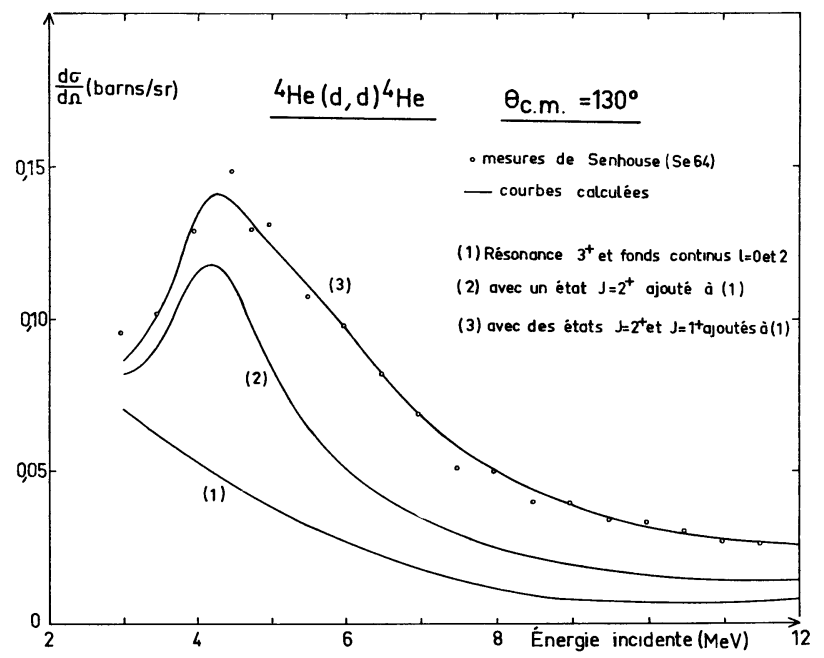

FIG. 2. - Ajustement des mesures expérimentales de ${ }^{4} \mathrm{He}(\mathrm{d}, \mathrm{d})^{4} \mathrm{He}$ par la courbe théorique calculée dans le formalisme de la matrice $S$. On montre la nécessité d'ajouter à la résonance $3^{+}$et aux fonds continus $l=0$ et $l=2$ (courbe 1 ) une résonance $2^{+}$(courbe 2 ), puis une résonance $1^{+}$(courbe 3 ). Les valeurs des paramètres sont données dans le tableau IV.

Nous avons alors ajouté la contribution d'un état résonnant $D^{2+}$ (courbe 2 ), puis d'un état résonnant $D^{1+}$ sans introduire de fonds continus correspondants. La courbe d'excitation à $130^{\circ}$ de la figure 2 et les distributions angulaires de la figure 3 ont été ajustées en supposant les contributions suivantes :

$$
\begin{array}{ll}
\text { fond } 1^{+}: l=0 \\
\text { niveau } 3^{+}: l=2 \\
\text { fond } 3^{+}: l=2 \\
\text { niveau } 2^{+}: l=2 \\
\text { niveau } 1^{+}: l=2 .
\end{array}
$$




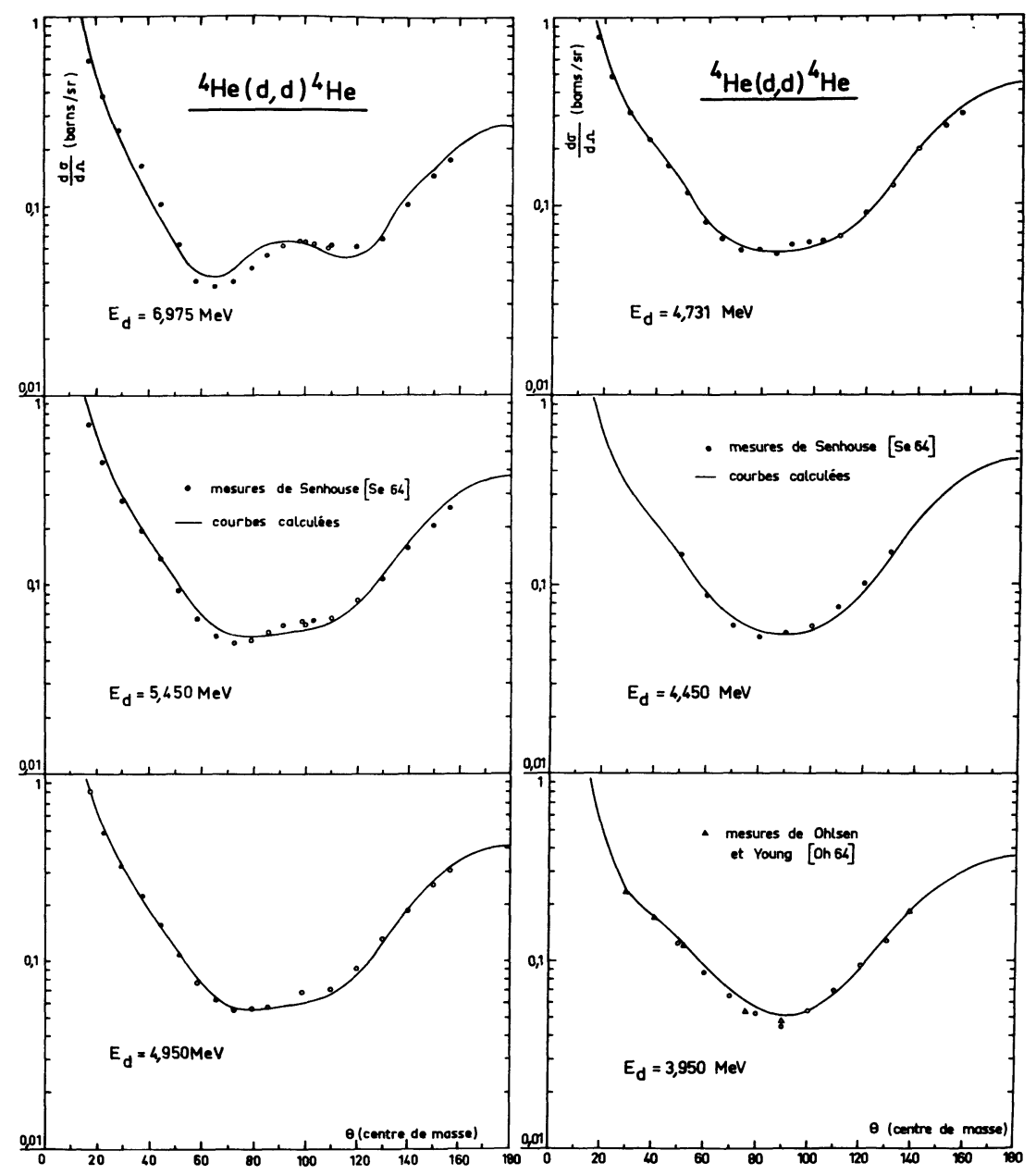

FIG. 3. - Interprétation des distributions angulaires de la diffusion résonnante ${ }^{4} \mathrm{He}+\mathrm{d}$ dans le formalisme de la matrice $S$. Les valeurs des paramètres obtenues sont données dans le tableau IV.

Les courbes calculées reproduisent bien les données expérimentales jusqu'à $9 \mathrm{MeV}$; au-delà de $9 \mathrm{MeV}$, l'accord est un peu moins bon en ce qui concerne l'intensité des minimums.

Les valeurs des paramètres obtenues sans introduire d'ondes $p$ non résonnantes sont données dans le tableau IV.

Les caractéristiques des états résonnants $D^{2+}$ et $D^{1+}$ sont comparées aux résultats antérieurs dans le tableau V.

3. Discussion. - Comme dans les interprétations précédentes, nous avons été amenés à introduire deux états résonnants $\left(2^{+}, T=0\right)$ et $\left(1^{+}, T=0\right)$ dans le lithium 6 mais dont l'énergie d'excitation est plus faible que celle trouvée en matrice $R$. La contribution de ces états à la section efficace de ${ }^{4} \mathrm{He}(\mathrm{d}, \mathrm{d})^{4} \mathrm{He}$ par unité d'énergie est faible; sur la figure 3 , le rapport des maximums à $4,5 \mathrm{MeV}$ et $1,070 \mathrm{MeV}$ est de $1 / 7$. Si ces états sont excités dans la réaction ${ }^{7} \mathrm{Li}\left({ }^{3} \mathrm{He}, \alpha\right)^{6} \mathrm{Li}$, on peut estimer en intégrant la fluctuation du fond qui apparaît sur les spectres entre 4 et $5 \mathrm{MeV}$ d'excitation, que leur contribution serait encore plus faible que celle de l'état $\left(T=0,3^{+}\right)$.

Pour expliquer ceci, la structuration des états considérés ne semble pas devoir être mise en cause. En effet, la largeur partielle $\Gamma_{d}$ est pratiquement égale à la largeur totale $\Gamma$ pour l'état $\left(3^{+}\right)$, tandis qu'elle est prépondérante par rapport aux largeurs partielles des autres processus pour les états $2^{+}$et $1^{+}$.

Une autre explication possible réside dans le mécanisme même de la réaction ${ }^{7} \mathrm{Li}\left({ }^{3} \mathrm{He}, \alpha\right){ }^{6} \mathrm{Li}$ qui défavoriserait les états très larges. Dans un mécanisme de « pick-up » par exemple, seuls apparaîtront les états dont la vie moyenne est supérieure à la durée d'interaction ${ }^{3} \mathrm{He}^{-} n$. Cette durée d'interaction, étant donné la barrière centrifuge, est supérieure à $10^{-21} \mathrm{~s}$, qui serait la durée d'une simple diffusion sur potentiel. Or la vie moyenne des états $2^{+}$et $1^{+}$est inférieure à $0,6 \times 10^{-21} \mathrm{~s}$, tandis que celle de l'état $3^{+}$est de 


\section{TABLEAU IV}

Paramètres déduits de l'analyse de la diffusion ${ }^{4} \mathrm{He}(\mathrm{d}, \mathrm{d})^{4} \mathrm{He}(b=4,5 \mathrm{fm})$ (toutes les valeurs sont données dans le système du centre de masse)

\begin{tabular}{|c|c|c|c|c|c|c|c|}
\hline & $E_{\text {rés }}(\mathrm{MeV})$ & $\Gamma(\underline{\mathrm{MeV}})$ & $Z_{\mathrm{c} n}$ & $\begin{array}{c}\xi_{\mathrm{c} n} \\
\text { (radian) } \\
-\end{array}$ & $R_{\mathbf{c} n}$ & $\begin{array}{c}\theta_{c n} \\
\text { (radian) } \\
-\end{array}$ & $\begin{array}{c}E_{\mathrm{exc}}{ }^{6} \mathrm{Li} \\
(\mathrm{MeV}) \\
-\end{array}$ \\
\hline $\begin{array}{l}\text { Fond } \\
\qquad \begin{array}{l}l=0 \\
J=1^{+}\end{array}\end{array}$ & & & & & 0,026 & 1,37 & \\
\hline $\begin{array}{c}\text { Résonance } \\
l=2 \\
J=3^{+} \\
\text {et fond } \\
l=2\end{array}$ & 0,71 & 0,0265 & 0,479 & 0,015 & 0,217 & 0,82 & 2,188 \\
\hline $\begin{array}{c}\text { Résonance } \\
l=2 \\
J=2^{+}\end{array}$ & 2,70 & 1,075 & 0,81 & $-0,22$ & & & 4,17 \\
\hline $\begin{array}{c}\text { Résonance } \\
l=2 \\
J=1^{+}\end{array}$ & 3,42 & 2,02 & 0,877 & $-0,28$ & & & 4,89 \\
\hline
\end{tabular}

TABLEAU V

Énergie D'excitation et LARgeur (en $\mathrm{MeV}$ ) Des États RÉsonNants $D^{2+}$ et $D^{1+}$ DE ${ }^{6} \mathrm{Li}$

\begin{tabular}{|c|c|c|c|c|c|}
\hline ÉtAT & $\begin{array}{c}\text { GALONSKY } \\
{[3]}\end{array}$ & $\begin{array}{c}\text { Senhouse } \\
{[11]}\end{array}$ & $\begin{array}{c}\text { McINTYRe } \\
{[12]}\end{array}$ & $\begin{array}{l}\text { MANI } \\
{[13]}\end{array}$ & $\begin{array}{l}\text { PRÉSENT } \\
\text { TRAVAIL }\end{array}$ \\
\hline- & - & - & - & - & - \\
\hline$D^{2+}$ & $4,52 \pm 0,08$ & 4,87 & $\begin{array}{c}4,6 \\
\Gamma \simeq 2,06\end{array}$ & 5,55 & $\begin{array}{c}4,17 \\
\Gamma \simeq 1,07\end{array}$ \\
\hline$D^{1^{+}}$ & 4,9 à 5,8 & 6,24 & $\begin{array}{c}5,7 \\
\Gamma \simeq 4,33\end{array}$ & 7,03 & 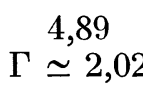 \\
\hline
\end{tabular}

l'ordre de $30 \times 10^{-21} \mathrm{~s}$. Il semble donc que l'hypothèse d'un mécanisme de « pick-up » puisse expliquer la non-excitation des états $\left(2^{+}, 0\right)$ et $\left(1^{+}, 0\right)$ par la réaction ${ }^{7} \mathrm{Li}\left({ }^{3} \mathrm{He}, \alpha\right){ }^{6} \mathrm{Li}$.

III. Mécanisme de la réaction ${ }^{7} \mathrm{Li}\left({ }^{3} \mathrm{He}, \alpha\right)^{6} \mathrm{Li}$. Les seules tentatives d'interprétation de la réaction ${ }^{7} \mathrm{Li}\left({ }^{3} \mathrm{He}, \alpha\right)^{6} \mathrm{Li}$ sont celles de Knudson et Wolicki d'une part [22], de Forsyth et Perry de l'autre [23].

Knudson et Wolicki ont ajusté la distribution angulaire à $4,5 \mathrm{MeV}$ du groupe $\alpha_{0}$ par un calcul en ondes planes du mélange de deux processus d'échange, « knock-out » et « heavy particle stripping ». Forsyth et Perry ont mesuré entre 0,8 et $5,5 \mathrm{MeV}$ la section efficace de la réaction ${ }^{7} \mathrm{Li}\left({ }^{3} \mathrm{He}, \alpha\right){ }^{6} \mathrm{Li}$ pour les groupes $\alpha(0), \alpha(2,18)$ et $\alpha(3,56)$. A partir d'un calcul en ondes planes, ils ont conclu, pour l'état fondamental, à des processus d'échange qui prennent le pas sur un processus de « pick-up » quand l'énergie incidente augmente, tandis que pour l'état $\left(3^{+}, 0\right)$ à $2,18 \mathrm{MeV}$ c'est le mécanisme de « pick-up » qui est prépondérant pour tout le domaine étudié.

Nous avons complété les mesures de Forsyth et Perry dans un domaine de 5 à $12 \mathrm{MeV}$ et avons cherché dans quelles limites un processus de « pick-up » peut ajuster ces résultats.

1. Procédure expérimentale. - Notre dispositif expérimental est décrit par ailleurs [24]. Les cibles et les détecteurs utilisés sont les mêmes que dans la première partie. L'intensité du faisceau ${ }^{3} \mathrm{He}^{++} \mathrm{du}$ Van de Graaff de $6 \mathrm{MeV}$ du G.R.N. de StrasbourgCronenbourg était de 0,03 à $0,05 \mu \mathrm{A}$. La charge intégrée par point était de $200 \mu \mathrm{C}$ pour les courbes d'excitation, de $300 \mu \mathrm{C}$ pour les distributions angulaires. Les taux de comptage ont été limités de manière à ce que le taux de perte dû au temps mort des analyseurs soit inférieur à $10 \%$ pour les angles vers l'avant. Une feuille mince de nickel absorbait les particules diffusées aux petits angles. Les impulsions de chacun 

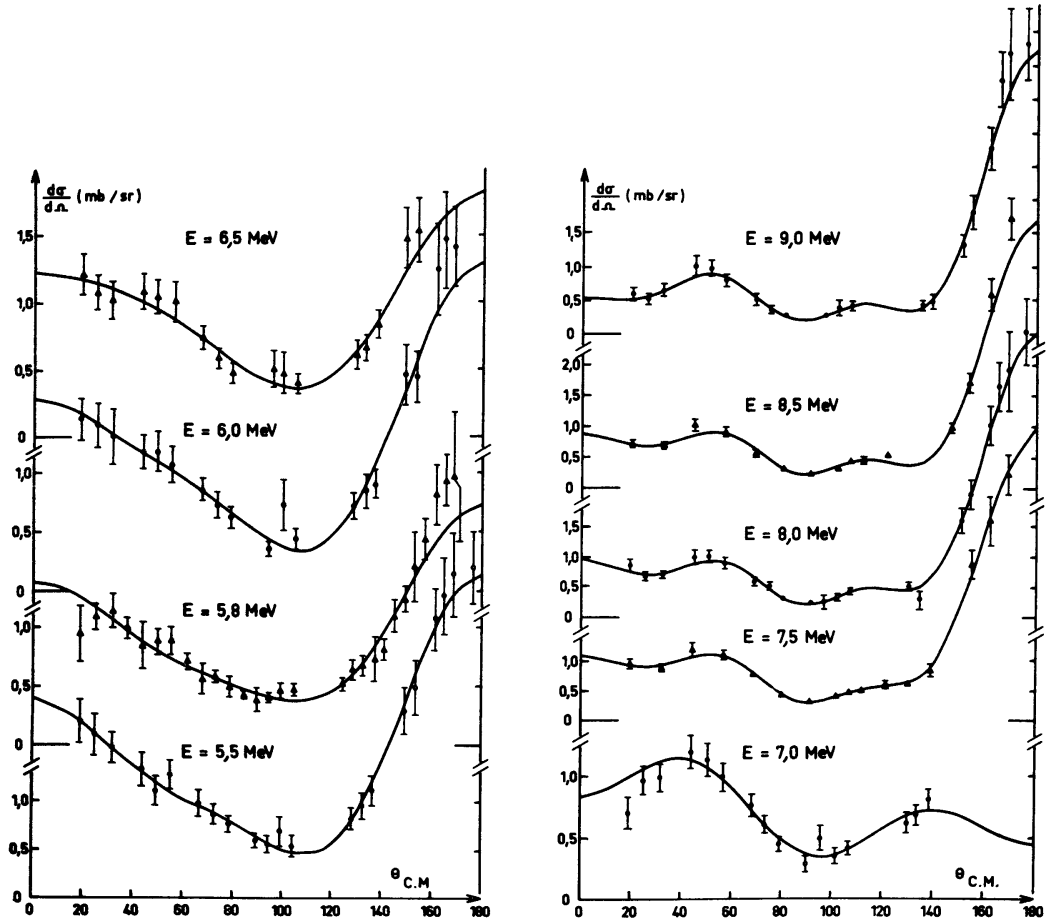

FIG. 4. - Distributions angulaires de la réaction ${ }^{7} \mathrm{Li}\left({ }^{3} \mathrm{He}, \alpha_{0}\right){ }^{6} \mathrm{Li}$

lissées par un développement en polynômes de Legendre. Niveau fondamental.

des détecteurs étaient distribuées sur un spectre de 512 canaux. Une des difficultés du traitement de ces spectres vient de l'ignorance de la forme des fonds sous-jacents aux groupes des particules $\alpha(2,18)$ et $\alpha(3,56 \mathrm{MeV})$, et à haute énergie, à celui des particules $\alpha(0)$. La délimitation des pics considérés entre pour une partie importante dans les erreurs reportées. Nous avons normalisé toutes nos mesures par rapport à celles de Forsyth et Perry.

2. Résultats expérimentaux. - Les courbes d'excitation pour l'état fondamental ont été mesurées à $35^{\circ}, 55^{\circ}, 75^{\circ}, 90^{\circ}, 125^{\circ}, 145^{\circ}$ et $165^{\circ}$ entre 5 et $12 \mathrm{MeV}$ par pas de $120 \mathrm{keV}$, sauf entre 7,8 et $8,8 \mathrm{MeV}$ où ce pas était de $30 \mathrm{keV}$. Le rendement tend à diminuer quand l'énergie augmente. L'allure monotone de ces courbes est en faveur d'un mécanisme par effets directs.

Les distributions angulaires ont été mesurées tous les $500 \mathrm{keV}$ entre 5 et $12 \mathrm{MeV}$ pour les trois premiers états de ${ }^{6} \mathrm{Li}$ et correspondent chacune à trois positions différentes de 7 détecteurs; le $8^{\mathrm{e}}$ fixé à $90^{\circ}$ a servi à les normaliser. Les mesures correspondant à l'état fondamental sont données figure 4 ; dans la figure 5 sont données celles correspondant à l'état à $2,18 \mathrm{MeV}$. Les courbes en traits pleins résultent du lissage des points expérimentaux par un développement en poly- nômes de Legendre dont l'ordre maximum est choisi par un test en $\chi^{2}$.

L'analogie de forme des distributions angulaires des groupes $\alpha(0)$ et $\alpha(3,56)$ est remarquable, en particulier le très fort rendement arrière dont l'importance augmente avec l'énergie. Un maximum faible subsiste dans la région de $40^{\circ}-60^{\circ}$ jusque vers $10 \mathrm{MeV}$ d'énergie incidente.

Les distributions angulaires pour le groupe $\alpha(2,18 \mathrm{MeV})$ changent peu en fonction de l'énergie incidente et présentent un maximum dans la région $40^{\circ}-60^{\circ}$, conformément à ce qu'avaient déjà observé Forsyth et Perry au-dessous de $5 \mathrm{MeV}$. Un maximum moins marqué apparaît vers $140^{\circ}$ à partir de $8,5 \mathrm{MeV}$.

3. INTERPRÉTATION EN D.W.B.A. - La section efficace du processus de « pick-up »a été calculée à l'aide du code Julie [25] avec l'approximation dite de portée nulle. Ce calcul nécessite la connaissance des paramètres optiques de la diffusion élastique dans les voies d'entrée et de sortie. Nous avons mesuré la distribution angulaire de ${ }^{7} \mathrm{Li}\left({ }^{3} \mathrm{He},{ }^{3} \mathrm{He}\right)^{7} \mathrm{Li}$ à $10 \mathrm{MeV}$ seulement, car il est très difficile de discriminer le pic élastique des nombreux pics des réactions compétitives. Nous avons d'autre part utilisé les mesures de Watters à $31,5 \mathrm{MeV}$ et de Grégoire et Macq à $23,8 \mathrm{MeV}$ sur la diffusion ${ }^{6} \mathrm{Li}(\alpha, \alpha){ }^{6} \mathrm{Li}[6]$. 

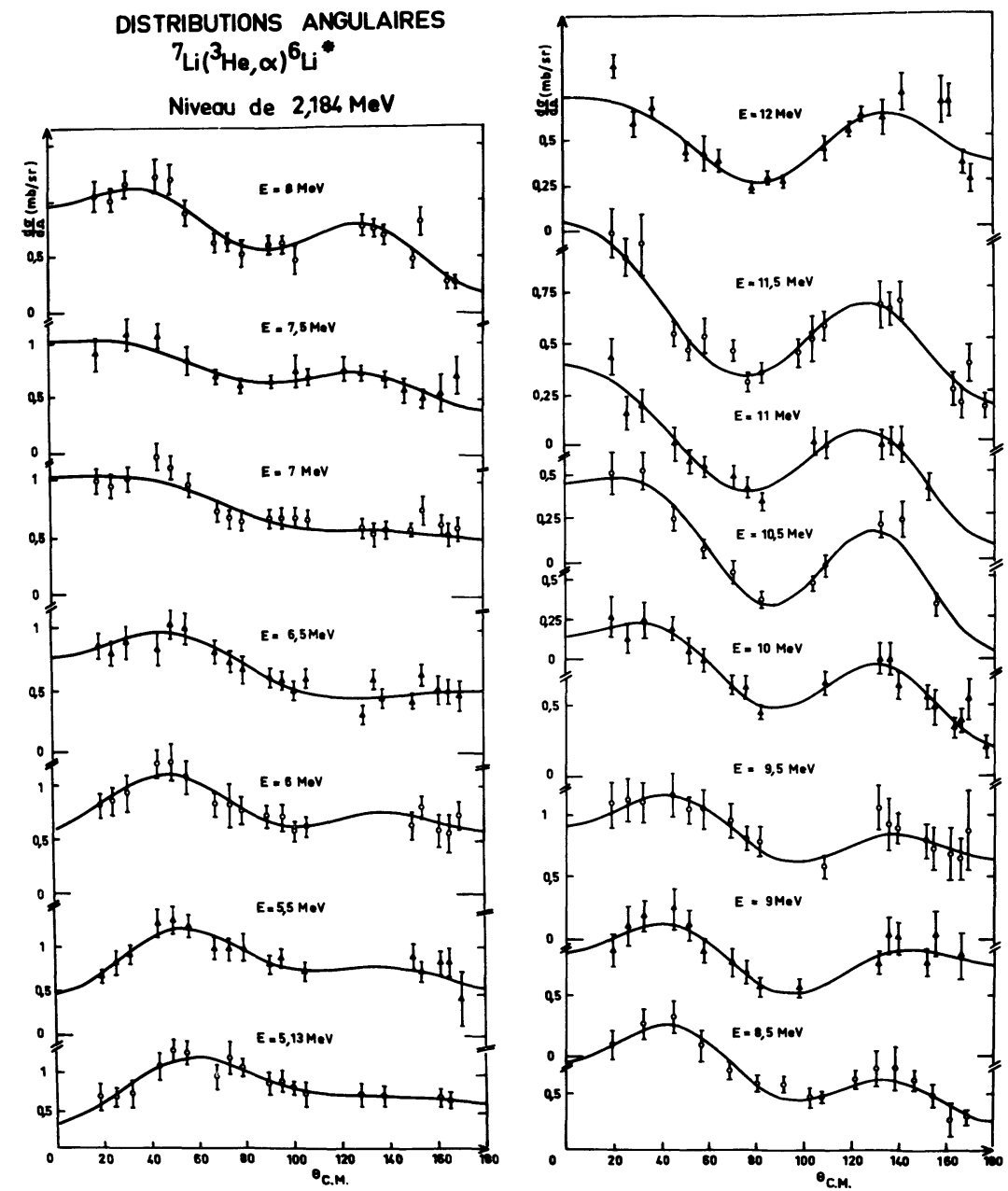

FIG. 5. - Distributions angulaires de la réaction ${ }^{7} \mathrm{Li}\left({ }^{3} \mathrm{He}, \alpha\right)^{6} \mathrm{Li}^{*}(2,184 \mathrm{MeV})$ lissées par un développement en polynômes de Legendre.

Le potentiel optique utilisé [26] est de la forme :

$$
\begin{aligned}
& V=-\frac{(U+i W)}{1+\exp \left(\frac{r-R_{\mathrm{r}}}{a}\right)} \\
&+ \begin{cases}\frac{z Z e^{2}}{2 R_{\mathrm{c}}}\left[3-\left(\frac{r}{R_{\mathrm{c}}}\right)^{2}\right] & \text { pour } r \leqslant R_{\mathrm{c}} \\
\frac{z Z e^{2}}{r} & \text { pour } r \geqslant R_{\mathrm{c}}\end{cases}
\end{aligned}
$$

où $R_{\mathrm{c}}$ est le rayon coulombien,

$U$ et $W$ les parties réelle et imaginaire du puits de potentiel,

$R_{\mathrm{r}}$ est le rayon d'interaction,

$a$ le paramètre de diffusion.

Nous n'avons pas cherché à inclure un potentiel spin-orbite, car une augmentation du nombre de paramètres n'a aucun sens physique dans une approxi- mation aussi grossière que la D.W.B.A. pour les noyaux légers.

Les jeux des paramètres qui nous ont donné le meilleur accord sont présentés dans le tableau VI.

Nous donnons les courbes de « pick-up » calculées en D.W.B.A. qui ajustent le mieux les données expérimentales figures 6,7 et 8 pour les groupes $\alpha(0)$, $\alpha(2,184 \mathrm{MeV})$ et $\alpha(3,56 \mathrm{MeV})$ respectivement.

Il serait nécessaire, pour parfaire cette analyse, de disposer de plus de données expérimentales pour les diffusions dans les voies d'entrée et de sortie de manière à mieux définir les paramètres optiques. Mais nous voyons dès à présent qu'un processus de « pick-up » ajuste bien les données expérimentales jusqu'à $100^{\circ}$ pour les trois groupes de particules $\alpha$ considérés. Cet accord devient moins bon à partir de $9 \mathrm{MeV}$ pour les groupes $\alpha(0)$ et $\alpha(3,56)$. La forte remontée arrière observée pour ces groupes et le maximum qui apparaît vers $140^{\circ}$ pour le groupe $\alpha(2,18)$ ne sont pas ajustés 
TABLEAU VI

\begin{tabular}{|c|c|c|c|c|c|c|}
\hline & Jeux & $\begin{array}{c}U(\mathrm{MeV}) \\
-\end{array}$ & $\begin{array}{c}W(\mathrm{MeV}) \\
-\end{array}$ & $R_{\mathrm{c}}($ fermi $)$ & $R_{\mathrm{r}}($ fermi) & $a$ (fermi) \\
\hline${ }^{7} \mathrm{Li}+{ }^{3} \mathrm{He}$ & $E 1$ & 63,923 & 17,064 & 2,7 & 3,135 & 0,559 \\
\hline à $10 \mathrm{MeV}$ & $E 3$ & 54,100 & 13,721 & 3,5 & 3,36 & 0,542 \\
\hline $\begin{array}{l}{ }^{6} \mathrm{Li}+\alpha \\
\quad \text { à } 23,8 \mathrm{MeV}\end{array}$ & $S 2$ & 48,870 & 11,173 & 2,183 & 3,54 & 0,513 \\
\hline $\begin{array}{l}\mathrm{Li}+\alpha \\
\text { à } 31,5 \mathrm{MeV}\end{array}$ & $S 3$ & 22,121 & 47,182 & 3,5 & 3,5 & 0,688 \\
\hline
\end{tabular}

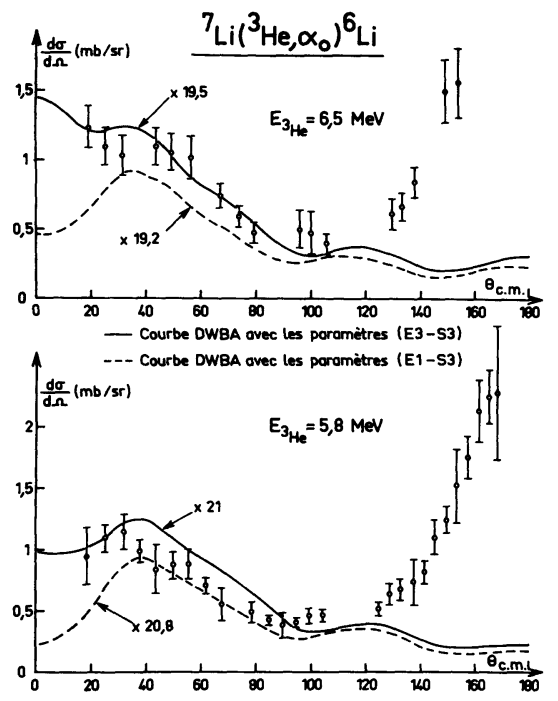

par les courbes de « pick-up». D'autres processus directs entrent probablement en compétition comme l'ont signalé les auteurs précédents [23], mais le calcul de ces processus nécessite des approximations inapplicables dans notre cas (approximation de masse infinie pour le cœur dans le cas du « knock-out»).

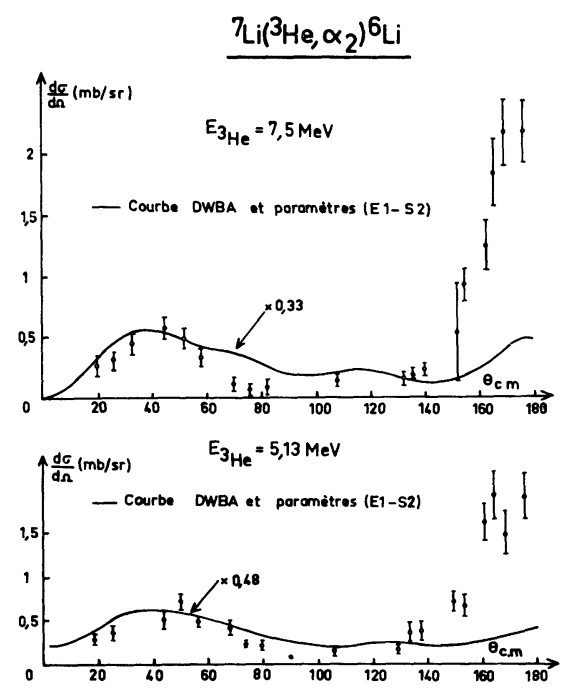

FIG. 6. - Interprétation de ${ }^{7} \mathrm{Li}\left({ }^{3} \mathrm{He}, \alpha_{0}\right){ }^{6} \mathrm{Li}$ par un processus de "pick-up " (code Julie). Les paramètres optiques utilisés sont donnés dans le tableau VI.

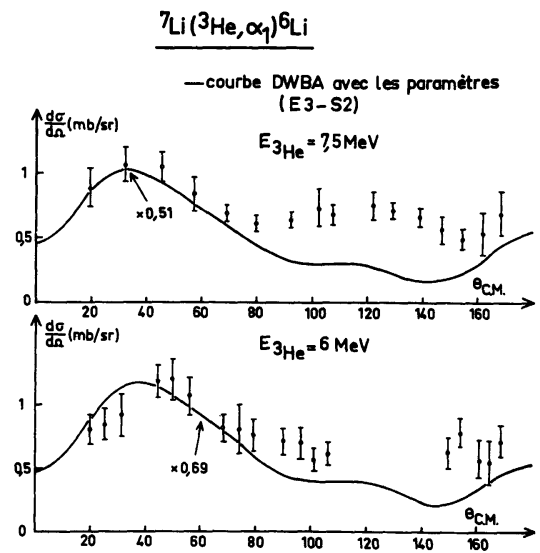

FIG. 7. - Interprétation de ${ }^{7} \mathrm{Li}\left({ }^{3} \mathrm{He}, \alpha\right)^{6} \mathrm{Li}^{*}(2,184 \mathrm{MeV})$ par un processus de " pick-up " (code Julie). Les paramètres optiques utilisés sont donnés dans le tableau VI.

IG. 8. - Interprétation de ${ }^{7} \mathrm{Li}\left({ }^{3} \mathrm{He}, \alpha\right)^{6} \mathrm{Li}^{*}(3,56 \mathrm{MeV})$ par un processus de "pick-up " (code Julie). Les paramètres optiques utilisés sont donnés dans le tableau VI.

Notre but était de vérifier si la réaction ${ }^{7} \mathrm{Li}\left({ }^{3} \mathrm{He}, \alpha{ }^{6} \mathrm{Li}\right.$ a un mécanisme de « pick-up ». Les résultats précédents montrent que c'est le cas à l'avant et au-dessous de $9 \mathrm{MeV}$, zone dans laquelle on avait le plus de chance, pratiquement, de pouvoir observer les états $D^{2+}$ et $D^{1+}$. Il semble donc que leur très faible section efficace de formation dans la réaction ${ }^{7} \mathrm{Li}\left({ }^{3} \mathrm{He}, \alpha\right){ }^{6} \mathrm{Li}$ soit bien en corrélation avec un mécanisme de « pick-up ».

Conclusion. - Nous donnons dans les trois premières colonnes du tableau VII les caractéristiques des 
TABLEAU VII

NiveAuX D'ÉNERGIE DE ${ }^{6} \mathrm{Li}$

Présent travail

Exc $(\mathrm{MeV})$
$\quad-$
0
2,188
$3,560(*)$
4,2
4,9
5,36

J $;$

Les quantités marquées $\left(^{*}\right)$ sont tirées de la littérature.

$\begin{array}{llc}J^{\pi} ; & T & \Gamma(\mathrm{keV}) \\ & - & - \\ 1^{+} ; & 0 & - \\ 3^{+} ; & 0 & 25 \\ 0^{+} ; & 1\left(^{*}\right) & <5\left({ }^{*}\right) \\ 2^{+} ; & 0 & \simeq 1000 \\ 1^{+} ; & 0 & \simeq 2000 \\ 2^{+}\left({ }^{*}\right) ; 1 & 540\end{array}$

INGLIS [1]
$(a / K=1,3)$
-
0
2,19
3,58
4,6
6,0
6,0

GOHEN

KURATH [1]

(en excluant

$\left.{ }^{6} \mathrm{Li}\right)$

0

1,4

2,0

3,6

6,2

4,8 six niveaux inférieurs de lithium 6 déduites de notre étude. Les seuls travaux de la littérature donnant trois états de ${ }^{6} \mathrm{Li}$ entre 4 et $5,4 \mathrm{MeV}$ sont ceux de Mani [7] par diffusion ( $\left.\mathrm{p}, \mathrm{p}^{\prime}\right)$ à $50 \mathrm{MeV}$ et ceux de Bouchez [8] par diffusion (n, $\left.\mathrm{n}^{\prime}\right)$ à $14 \mathrm{MeV}$. Les autres réactions n'ont pas été analysées par des méthodes suffisamment résolvantes ou bien sont faites à basse énergie ou sont aussi des réactions de " pick-up ».

Nous comparons nos résultats avec les prévisions théoriques d'Inglis [1] et de Cohen et Kurath [1] (tableau VII).

L'accord de nos résultats avec les valeurs de Cohen et Kurath est beaucoup moins bon qu'avec celles plus anciennes d'Inglis dont le modèle très élémentaire d'un couplage LS presque pur $(a / K \simeq 1,3)$ donne la même suite de niveaux que celle que nous avons obtenue.

Il est remarquable de constater que c'est le modèle plus ancien et élémentaire d'Inglis qui rend le mieux compte de nos résultats, en supposant dans le lithium 6 un couplage LS presque pur.

Remerciements. - Nous remercions tous ceux qui nous ont aidés dans la réalisation de ce travail, en particulier les Docteurs Bock et Martin du MaxPlanck Institute de Heidelberg pour le code Julie, et le Docteur Fridman du Département de Physique Corpusculaire de Strasbourg-Cronenbourg pour l'accès à l'ordinateur.

\section{BIBLIOGRAPHIE}

[1] Inglis (D. R.), Phys. Rev., 1952, 87, 915.

Cohen (S.) et Kurath (O.), Nucl. Phys., 1965, 73, 1.

Barker (F. C.), Nucl. Phys., 1966, 83, 418.

Goldhammer, Hili, et Nachamkin, Nucl. Phys., 1968, A 106, 62.

[2] AJZENBERG-Selove (F.) et LAURiTSEN (T.), Nucl. Phys., 1959, 11, 1.

LAURiTSEN (T.) et AJZENBERG-Selove (F.), Nucl. Data Sheets, may $1952 ;$ Nucl. Phys., 1966, 78, 1.

[3] Allen, Almovist et Bigham, Phys. Rev., 1965, 99, 631 A ; Proc. Phys. Soc., 1960, 75, 913.

[4] LINCK (J.), Thèse de $3^{\mathrm{e}}$ cycle, Strasbourg, 1962.

LINCK (J.), NICOLAS-LINCK (I.), BIIWES (R.) et MAgnaC-VALETTLE (D.), J. Physique, 1963, 24, 983.

[5] Cocke (L.), Nucl. Phys., 1968, A 110, 321.

[6] Watters, Phys. Rev., 1956, 103, 1763.

GrÉGOIre et MacQ, Phys. Lett., 1964, 8, 2614.
[7] Browne et Bockelmann, Phys. Rev., 1957, 105, 1301.

Hasselgrein et collaborateurs, Nucl. Phys., 1965, $69,81$.

ManI (G. S.) et Dix (A. D. B.), Nucl. Phys., 1968, A 106, 251.

[8] Bouchez (R.) et collaborateurs, C. R. Acad. Sc., 1965, 260, 3922.

[9] Groce et Whaling, Phys. Rev., 1963, 132, 2614.

[10] KulL, Phys. Rev., 1967, 163, 1066.

Hamburger et Cameron, Phys. Rev., 1960, 117, 781.

LEVINE, Beinder et MCGruer, Phys. Rev., 1955, 97, 1249 .

[11] Galonsky et collaborateurs, Phys. Rev., 1955, 98, 586.

Galonsky et McElilistrem, Phys. Rev., 1955, 98, 590.

[12] Senhouse et Tombreilio, Nucl. Phys., 1964, 57, 624. MCInTYRe et HAEBerLI, Nucl. Phys., 1967, A 91, 382. 
[13] Mani et Tarrats, Nucl. Phys., 1968, A 107, 624.

[14] AJZENBERG-SELOVE (F.), WATSON et MIDDLETON, Phys. Rev., 1965, 139, B 592.

[15] LINCK (I.), LINCK (J.), SeLTZ (R.) et MagnaCVALETTte (D.), J. Physique, 1966, C 1, 59.

[16] RayeT (M.), Service de Phys. Nucl. et de Métrologie Nucl., Bruxelles, Bulletin no 15 .

[17] Wakefield (B.) et MACEFIELd (B.), Nucl. Phys., $1968,114,561$.

[18] Ohisen (G. G.) et Young (P. G.), Nucl. Phys., $1964,52,134$

[19] Kraus (L.), Linck (I.) et MagnaC-ValetTte (D.), Nucl. Phys. (à paraître).

[20] Humbleit (J.), Nucl. Phys., 1964, 50, 1.
[21] Hooke et JEEvEs, J. Assoc. of Computing Machinery, 1962, 8, 212.

[22] KNUDSON et WoLICKI, " Direct Nuclear Interactions ", Padoue, 1963, 981.

[23] Forsyth (P. D.) et Perry (R. R.), Nucl. Phys., 1965, 67, 517 .

[24] SELTZ (R.), Thèse de doctorat d’État, Strasbourg, 1967.

[25] BASSEL (R. H.), DRISko (R. M.) et SATChLER (G. R.), ORNL 3240.

[26] Thompson (W. S.) et GILLE (E.), Technical Report no 9 "OPTIX 1 ", Florida State University.

[27] Lane et Thomas, Rev. Mod. Phys., 1958, 30, 257. 